\title{
Loop exponent in DNA bubble dynamics
}

\author{
Vojtěch Kaiser ${ }^{1,2,3, *}$ and Tomáš Novotný ${ }^{1, \#}$ \\ ${ }^{1}$ Department of Condensed Matter Physics, Faculty of Mathematics and Physics, \\ Charles University in Prague, Ke Karlovu 5, 12116 Prague, Czech Republic \\ ${ }^{2}$ Laboratoire de Physique, École Normale Supérieure de Lyon, Université de Lyon, \\ 46 Allée d'Italie, 69364 Lyon Cedex 07, France \\ ${ }^{3}$ Max-Planck-Institut für Physik komplexer Systeme, Nöthnitzer Straße 38, 01187 \\ Dresden, Germany \\ E-mail: *vojtech.kaiser@ens-lyon.fr; \#tno@karlov.mff.cuni.cz
}

\begin{abstract}
Dynamics of DNA bubbles are of interest for both statistical physics and biology. We present exact solutions to the Fokker-Planck equation governing bubble dynamics in the presence of a long-range entropic interaction. The complete meeting time and meeting position probability distributions are derived from the solutions. Probability distribution functions reflect the value of the loop exponent of the entropic interaction. Our results extend previous results which concentrated mainly on the tails of the probability distribution functions and open a way to determining the strength of the entropic interaction experimentally which has been a matter of recent discussions. Using numerical integration, we also discuss the influence of the finite size of a DNA chain on the bubble dynamics. Analogous results are obtained also for the case of subdiffusive dynamics of a DNA bubble in a heteropolymer, revealing highly universal asymptotics of meeting time and position probability functions.

PACS numbers: 05.10.Gg, 82.37.j, 87.15.v, 87.14.gk
\end{abstract}

Submitted to: J. Phys. A: Math. Gen. 


\section{Introduction}

DNA bubbles are local openings of the DNA double-helix caused by thermal or torsional forces. The genetic information is stored inside of the double-helix. Hence, the bubbles facilitate binding proteins to DNA for transcription, replication, and repair [1. Bubbles (also known as loops) influence DNA thermodynamics and mediate longrange interactions along the DNA chain necessary for the existence of the melting phase transition [2].

DNA undergoes a melting (denaturation) transition during which the double helix separates into two single strands. The nature of the DNA melting transition was first described by Poland and Scheraga [3, 4]. Their theory assumes that a DNA molecule consists of loops and bound double-stranded segments. The Poland-Scheraga model is a basic yet extendable model of DNA; it constitutes a limiting case of more complex models which motivates our study of its dynamics.

In the Poland-Scheraga model, whose form of the Gibbs free energy we use in this work, the strength of the long-range interactions and thus the order of the phase transition is determined by the loop entropy exponent $c$ multiplying the logarithmic potential term. The value of $c$ depends on the self-avoiding and mutually avoiding properties of DNA bubbles. Diffusion in a logarithmic potential has been the subject of recent studies [5, 6].

Previous works studied the bubble dynamics in DNA denaturation using a sequenceaveraged continuous model [7, 8, 9, 10, 11]. Extending this model, we aim to show how the value of the loop exponent could be determined from bubble-closing dynamics. We focus on the physical aspects of the dynamics and do not explicitly discuss biological processes involving DNA bubbles in this work, because recent studies have shown them to be strongly sequence-dependent [12, 13, 14, and controlled by the interplay between torsional and thermal forces [15, 16, 17, 18, 19].

Fisher [20] obtained $c=1.76$ considering only excluded-volume interactions among bases in the same bubble, corresponding to a continuous phase transition. This approximation is valid for short chains ( $\sim 10^{4}$ base pairs) [7]. If inter-bubble and interchain interactions are included as well, a calculation based on the polymer network theory predicts $c=2.12$ in the thermodynamic limit, yielding a first-order phase transition [21] (see Fig. 11 for an illustration of the excluded-volume interactions).

The value of the loop entropy exponent has not yet been conclusively measured due to its strong correlation with the cooperativity parameter $\sigma$ [22]. Therefore, we study how $c$ influences DNA bubble closing dynamics, which are independent of $\sigma$. Previous results [9, 10, 11, 23] focused mainly on the long-time asymptotics where we expect the experimental signal-to-noise ratio to be low. The solution presented below is valid for all times. Moreover, we also find the solution for the meeting position, which especially around its peak proves as a useful complementary quantity for experimental verification.

We study two regimes of bubble dynamics - diffusive and subdiffusive - shown to exist for a random DNA sequence [24]. The subdiffusive regime appears in the 


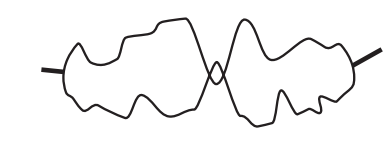

a

Figure 1. Loop entropy coefficient $c$ dependence on the excluded volume interactions taken into account. a) No exclusion effects, $c=3 / 2$ comes purely from the constraint on the bubble to form a closed loop [4. b) Excluded volume within a single bubble, $c=1.76$ given by the statistics of 3D self-avoiding walks 20 . c) Excluded volume among all segments of the DNA chain, renormalized value is $c=2.12[21$.

continuous limit of bubble trapping in AT rich regions, whose melting is energetically less costly than GC-region melting. For both of these regimes, we compute the probability distribution functions (PDFs) of the meeting time, i.e., the time when the bubble ends meet and the bubble closes, and the meeting position PDFs. The computations are carried out analytically in the Laplace picture and numerically inverse transformed to the time domain afterwards.

In the diffusive regime, we show how the loop exponent influences bubble dynamics in a finite DNA chain. Our result extends previous results for finite molecules that neglected the loop entropy [25, 26, 27]. Meeting time and meeting position PDFs are obtained using the finite-element method.

The article is organised as follows. Section 2 details our model. Section 3 solves the diffusive model, while section 4 the subdiffusive one. In section 5, we describe bubble diffusion along finite DNA chains. In section [6, we briefly comment on the experimental feasibility of verifying the obtained results based on known and estimated thermodynamic parameters of DNA. We close with concluding section 7 .

\section{Model definition}

The Poland-Scheraga model describes the DNA molecule as a one-dimensional chain of alternating parts: loops and bound segments [4, 28]. Initiating a loop and denaturing a subsequent base pair are associated with their Gibbs free energy $G_{\text {init }}$ and $\Delta G_{\mathrm{bp}}$, respectively. The value of $\Delta G_{\mathrm{bp}}$ is usually determined by melting small DNA molecules [29] and corresponds to a complete denaturation of the DNA chain. A closed loop can attain fewer configurations than two fully denatured strands. The difference in allowed configurations scales as a power of the number of open base pairs in the loop with the loop entropy exponent $c$. The entropy reduction is thus logarithmic in the bubble size: $\Delta S_{\text {loop }}=-k_{\mathrm{B}} c \ln n$.

The dependence of entropy on the loop length mediates an effective long-range interaction between the ends of the loop where the opening and closing dynamics take place. Because the entropies of individual loops contribute to the total Gibbs energy 
$G=H-T S$ of the DNA molecule, the system interacts on a long range, which is a necessary condition for a phase transition to occur in one dimension [30]. The long-range interactions grow more prominent with increasing $c$. The transition occurs if $c>1$ and is of the first order for $c>2$.

\subsection{Gibbs free energy landscape}

Initiating a loop costs a substantial amount of energy $\left(G_{\text {init }} \simeq 10 k_{\mathrm{B}} T_{\text {phys }}\right.$ at physiological temperatures $T_{\text {phys }} \simeq 310 \mathrm{~K}$ ). The statistical weight of opening the bubble $\sigma(T)=$ $\exp \left(-\beta G_{\text {init }}\right)$ is called the cooperativity parameter; its typical value is $\sigma\left(T_{\text {phys }}\right) \simeq 10^{-4}$. Bubbles are thus separate thermal excitations except close to the melting temperature $T_{\mathrm{c}}$ [7]. Treating a single bubble approximates the DNA chain dynamics well.

We approximate the number of open base pairs $n$ by a continuous variable $x \geq 0$. The Gibbs free energy for a single bubble with $x$ open base pairs depicted in Figure 2 then reads:

$$
G(x)=G_{\text {init }}+x \Delta G_{\mathrm{bp}}+k_{\mathrm{B}} T c \ln x .
$$

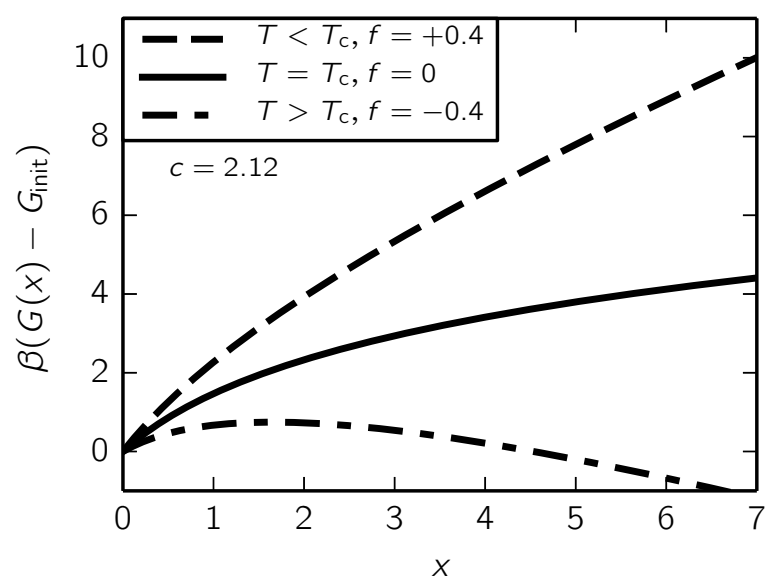

Figure 2. Character of the Gibbs free energy (in units of $k_{\mathrm{B}} T$ ) as a function of the number of open base pairs for various temperatures. Under the melting temperature $T_{\mathrm{c}}$, both the linear term $f x=\beta \Delta G_{b p} x / 2$ and the logarithmic term $c / x$ contribute to the closing of the bubble. At the phase transition, only the logarithmic term remains. Above $T_{\mathrm{c}}$, the logarithmic term serves as a nucleation barrier to the denaturation of DNA.

\subsection{Two types of DNA bubble dynamics}

Bubbles in a random (spatially uncorrelated) DNA sequence have two regimes of propagation separated by a glass transition: diffusive and subdiffusive [24]. Below the glass transition $\left(T \leq T_{\mathrm{g}} \leq T_{\mathrm{c}}\right.$ ), the dynamics of bubbles are diffusive. Between $T_{\mathrm{g}}$ and $T_{\mathrm{c}}$, the subdiffusive regime appears with an exponent of subdiffusion $0<\nu<1$ monotonously decreasing from unity at $T_{\mathrm{g}}$ to zero at $T_{\mathrm{c}}$. 
We consider only the closing dynamics of a single bubble in the following, due to the low statistical weight $\sigma(T) \ll 1$ for reopening of the bubble. The dynamics being dominated by loop closing was observed experimentally in [8].

\section{Diffusive dynamics}

\subsection{Bubble-size Fokker-Planck equation}

In the continuous approximation, the Fokker-Planck equation (FPE) describes the diffusive dynamics of a bubble. Given a potential $G$, FPE has the form [31, Sec. 5.4]:

$\partial_{\tau} P_{\mathrm{s}}\left(x, \tau \mid x_{0}\right)=-\partial_{x} S_{\mathrm{s}}\left(x, \tau \mid x_{0}\right)=K \partial_{x}\left[\partial_{x} P_{\mathrm{s}}\left(x, \tau \mid x_{0}\right)+\beta P_{\mathrm{s}}\left(x, \tau \mid x_{0}\right) \partial_{x} G(x)\right]$,

where $P_{\mathrm{s}}\left(x, \tau \mid x_{0}\right)$ is the bubble-size PDF $₫$ at time $\tau, S_{\mathrm{s}}\left(x, \tau \mid x_{0}\right)$ the probability current, $K$ the diffusion constant, and $\beta$ the inverse temperature. Initially, $x_{0}$ base pairs are open and $P_{\mathrm{s}}\left(x, t=0 \mid x_{0}\right)=\delta\left(x-x_{0}\right)$. We introduce dimensionless time $t=\tau K / 2$, where $K$ can be regarded as a fitting parameter for comparison with the experiment. The definition of dimensionless time $t$ reflects that the bubble shrinks or grows at its edges, which propagate with diffusion constant $K / 2$.

The potential (2.1) introduces a drift term opening or closing the bubble

$$
\partial_{x} G(x)=\Delta G_{b p}+k_{B} T c / x .
$$

Substituting (3.2) into (3.1) yields

$$
\partial_{t} P_{\mathrm{s}}\left(x, t \mid x_{0}\right)-2 \partial_{x x} P_{\mathrm{s}}\left(x, t \mid x_{0}\right)-4 \partial_{x}\left[\left(f+\frac{\gamma}{x}\right) P_{\mathrm{s}}\left(x, t \mid x_{0}\right)\right]=0,
$$

where $f \equiv \beta \Delta G_{b p} / 2$ and $\gamma \equiv c / 2$ are introduced. The absorbing boundary condition $\lim _{x \rightarrow 0+} P_{\mathrm{s}}\left(x, t \mid x_{0}\right)=0$ represents closing dynamics. We focus on the regime $T<T_{\mathrm{c}}$, where $f>0$.

\subsection{Mapping to the Coulomb problem}

FPE (3.3) maps to the imaginary-time Coulomb problem [9, 10]. This mapping is an example of transforming the Fokker-Planck operator to a Hermitian one [31]. The transformed PDF $w\left(x, t \mid x_{0}\right)$ is related to the original bubble size PDF by

$$
P_{\mathrm{s}}\left(x, t \mid x_{0}\right)=e^{-f\left(x-x_{0}\right)}\left(\frac{x}{x_{0}}\right)^{-\gamma} w\left(x, t \mid x_{0}\right) .
$$

The spatial part of FPE (3.3) is then Hermitian

$$
\begin{aligned}
& {\left[-\partial_{t}+2 \partial_{x x}-2 f^{2}-\frac{4 f \gamma}{x}-\frac{2 \gamma(\gamma+1)}{x^{2}}\right] w\left(x, t \mid x_{0}\right)=0} \\
& w\left(x, t \rightarrow 0^{+} \mid x_{0}\right)=\delta\left(x-x_{0}\right), \quad w\left(x \rightarrow 0^{+}, t \mid x_{0}\right)=0 .
\end{aligned}
$$

Fogedby and Metzler used this transform to obtain the spectrum, the long-time asymptotics of bubble dynamics, as well as the complete solution at the critical point where $f=0[9,10]$.

$\ddagger$ In the text we use $P_{\mathrm{s}}, P_{\mathrm{c}}$, and $P_{\mathrm{e}}$ to denote the bubble size, centre, and edge PDFs, respectively. 


\subsection{Green function}

While the spectral approach to (3.5) provides an insight to the long-time behaviour of bubble dynamics, summing the eigenfunctions to obtain the short-time dynamics proves to be difficult. To obtain an exact Laplace picture form of the solution (denoted by the bar over the quantities), we take a Laplace transform of (3.5) instead:

$$
\begin{gathered}
{\left[\partial_{x x}-\left(f^{2}+\frac{s}{2}\right)-\frac{2 f \gamma}{x}-\frac{\gamma(\gamma+1)}{x^{2}}\right] \bar{w}\left(x, s \mid x_{0}\right)=-\frac{1}{2} \delta\left(x-x_{0}\right),} \\
\bar{w}\left(x \rightarrow 0^{+}, s \mid x_{0}\right)=0 .
\end{gathered}
$$

The task is to find the Green function of a differential operator with $s$ as a parameter. We find homogeneous solutions of (3.6) and construct the Green function as their linear combination.

The homogeneous solutions are the Whittaker functions $M_{(-f \gamma / \alpha(s) ; \gamma+1 / 2)}(2 x \alpha(s))$ and $W_{(-f \gamma / \alpha(s) ; \gamma+1 / 2)}(2 x \alpha(s))$, where we defined $\alpha^{2}(s)=f^{2}+s / 2 . M_{(-f \gamma / \alpha(s) ; \gamma+1 / 2)}(2 x \alpha(s))$ is regular for $x \rightarrow 0$ and diverges at infinity; $W_{(-f \gamma / \alpha(s) ; \gamma+1 / 2)}(2 x \alpha(s))$ is a regular solution at infinity and singular at the origin [32].

The Green function is continuous and has a first-derivative discontinuity $\lim _{x \rightarrow x_{0}+} \partial_{x} \bar{w}\left(x, s \mid x_{0}\right)-\lim _{x \rightarrow x_{0}-} \partial_{x} \bar{w}\left(x, s \mid x_{0}\right)=-1 / 2$. The linear combination of Whittaker functions that satisfies both (3.6) and the above conditions is

$$
\begin{aligned}
\bar{w}\left(x, s \mid x_{0}\right)= & \frac{\Gamma\left(1+\gamma+\frac{f \gamma}{\alpha(s)}\right)}{4 \alpha(s) \Gamma(2+2 \gamma)}\left[\theta\left(x-x_{0}\right) M_{\left(-\frac{f \gamma}{\alpha(s)} ; \gamma+\frac{1}{2}\right)}\left(2 x_{0} \alpha(s)\right) W_{\left(-\frac{f \gamma}{\alpha(s)} ; \gamma+\frac{1}{2}\right)}(2 x \alpha(s))+\right. \\
& \left.+\theta\left(x_{0}-x\right) W_{\left(-\frac{f \gamma}{\alpha(s)} ; \gamma+\frac{1}{2}\right)}\left(2 x_{0} \alpha(s)\right) M_{\left(-\frac{f \gamma}{\alpha(s)} ; \gamma+\frac{1}{2}\right)}(2 x \alpha(s))\right]
\end{aligned}
$$

where the prefactor ensures the correct discontinuity of the first derivative at $x_{0}$ and can be related to the constant Wronskian of the Whittaker functions [32, Sec. 13.14.26]

$$
\mathcal{W} \equiv M_{\kappa ; \mu}(x) \frac{d}{d x} W_{\kappa ; \mu}(x)-W_{\kappa ; \mu}(x) \frac{d}{d x} M_{\kappa ; \mu}(x)=-\frac{\Gamma(1+2 \mu)}{\Gamma\left(\frac{1}{2}+\mu-\kappa\right)} .
$$

The Green function of (3.3) in the Laplace picture is obtained by substitution of $\bar{w}\left(x, s \mid x_{0}\right)$ into (3.4)

$$
\begin{aligned}
\bar{P}_{\mathrm{s}}\left(x, s \mid x_{0}\right)= & e^{-f\left(x-x_{0}\right)}\left(\frac{x}{x_{0}}\right)^{-\gamma} \frac{\Gamma\left(1+\gamma+\frac{f \gamma}{\alpha(s)}\right)}{4 \alpha(s) \Gamma(2+2 \gamma)} \times \\
& \times\left[\theta\left(x-x_{0}\right) W_{\left(-\frac{f \gamma}{\alpha(s)} ; \gamma+\frac{1}{2}\right)}(2 x \alpha(s)) M_{\left(-\frac{f \gamma}{\alpha(s)} ; \gamma+\frac{1}{2}\right)}\left(2 x_{0} \alpha(s)\right)+\right. \\
& \left.+\theta\left(x_{0}-x\right) M_{\left(-\frac{f \gamma}{\alpha(s)} ; \gamma+\frac{1}{2}\right)}(2 x \alpha(s)) W_{\left(-\frac{f \gamma}{\alpha(s)} ; \gamma+\frac{1}{2}\right)}\left(2 x_{0} \alpha(s)\right)\right] .
\end{aligned}
$$

To our best knowledge, an analytical form of the inverse Laplace transform of the previous equation does not exist and has to be evaluated numerically instead. Current experimental techniques cannot directly observe time dependence of the bubble-size PDF. Therefore, it is necessary to evaluate derived PDFs, namely for the meeting time and meeting position. In the following, we give the Laplace picture expressions for these PDFs. 


\subsection{Meeting time PDF}

The probability of the bubble surviving up to time $t$ is the total integral of the $P_{\mathrm{s}}\left(x, t \mid x_{0}\right)$ over all possible $x$. This can only be reduced by the probability flux out of the system at its boundaries [33, 27, 34], which for our case happens if the two edges of a bubble meet. Thus, we define the meeting time PDF $\bar{\pi}_{\mathrm{mt}}\left(t \mid x_{0}\right)$ as the probability current through the absorbing boundary at the origin

$$
\pi_{\mathrm{mt}}\left(t \mid x_{0}\right) \equiv-S_{\mathrm{s}}\left(0, t \mid x_{0}\right)=\lim _{x \rightarrow 0}\left[2 \partial_{x}+4\left(\frac{\gamma}{x}+f\right)\right] P_{\mathrm{s}}\left(x, t \mid x_{0}\right)
$$

After substituting from (3.9) into the previous equation, the Laplace transform of the meeting time PDF reads

$$
\begin{aligned}
\bar{\pi}_{\mathrm{mt}}\left(s \mid x_{0}\right)= & \frac{e^{f x_{0}}}{\Gamma(1+2 \gamma)}\left(x_{0} \sqrt{4 f^{2}+2 s}\right)^{\gamma} \Gamma\left(1+\gamma+f \gamma \sqrt{\frac{2}{2 f^{2}+s}}\right) \times \\
& \times W_{\left(-f \gamma \sqrt{\frac{2}{2 f^{2}+s}} ; \gamma+\frac{1}{2}\right)}\left(x_{0} \sqrt{4 f^{2}+2 s}\right) .
\end{aligned}
$$

We invert the Laplace transform numerically. Generally, the numerical inverse Laplace transform is ill-conditioned because the associated operator is unbounded [35]. Available numerical methods restrict the operator to smaller spaces of functions. A class of these methods invert functions with known pole structure, while other methods operate on real axis both in the direct and Laplace picture (see Refs. [35, 36] for an overview).

We used the Stehfest algorithm [37] in this work which computes the Laplace inverse as a weighted sum of $\bar{\pi}_{\mathrm{mt}}\left(s \mid x_{0}\right)$ for real $s$. The algorithm requires the resulting functions to be continuous with bounded derivatives. The bounds of derivatives determine the stability of the algorithm. In our case, the selected parameters result in curves with sufficiently small derivatives. To further avoid instabilities, we used high-precision arithmetics (up to 100 digits).

The results are shown in Figure 3 together with the asymptotics

$$
\pi_{\mathrm{mt}} \propto t^{-(c+3) / 2} \exp \left(-2 f^{2} t\right)
$$

from Ref. [9]. Both parameters $c$ and $f$ influence strongly not only the tails of the PDFs but also their peak values \&

\subsection{Meeting position PDF}

Apart from changing its size, a bubble diffuses freely along the DNA chain, described by the bubble centre position $y$ and its $\mathrm{PDF} P_{\mathrm{c}}\left(y, t \mid y_{0}\right)$. The energy landscape is flat because pairing energies are sequence averaged. The Fokker-Planck equation for the centre position reads

$$
\partial_{t} P_{\mathrm{c}}\left(y, t \mid y_{0}\right)=\partial_{y y} P_{\mathrm{c}}\left(y, t \mid y_{0}\right)
$$

$\S$ The values in the figures correspond to an ideal loop $(c=1.5)$, self-avoiding loop $(c=1.76)$ [20], renormalised value in the presence of an external stretching force $(c=1.85)$ [38, 39, self-avoiding and mutually avoiding loops $(c=2.12)$ [21], and the maximal allowed value for RNA with pseudoknots and hairpins $(c=2.49)[40$. 

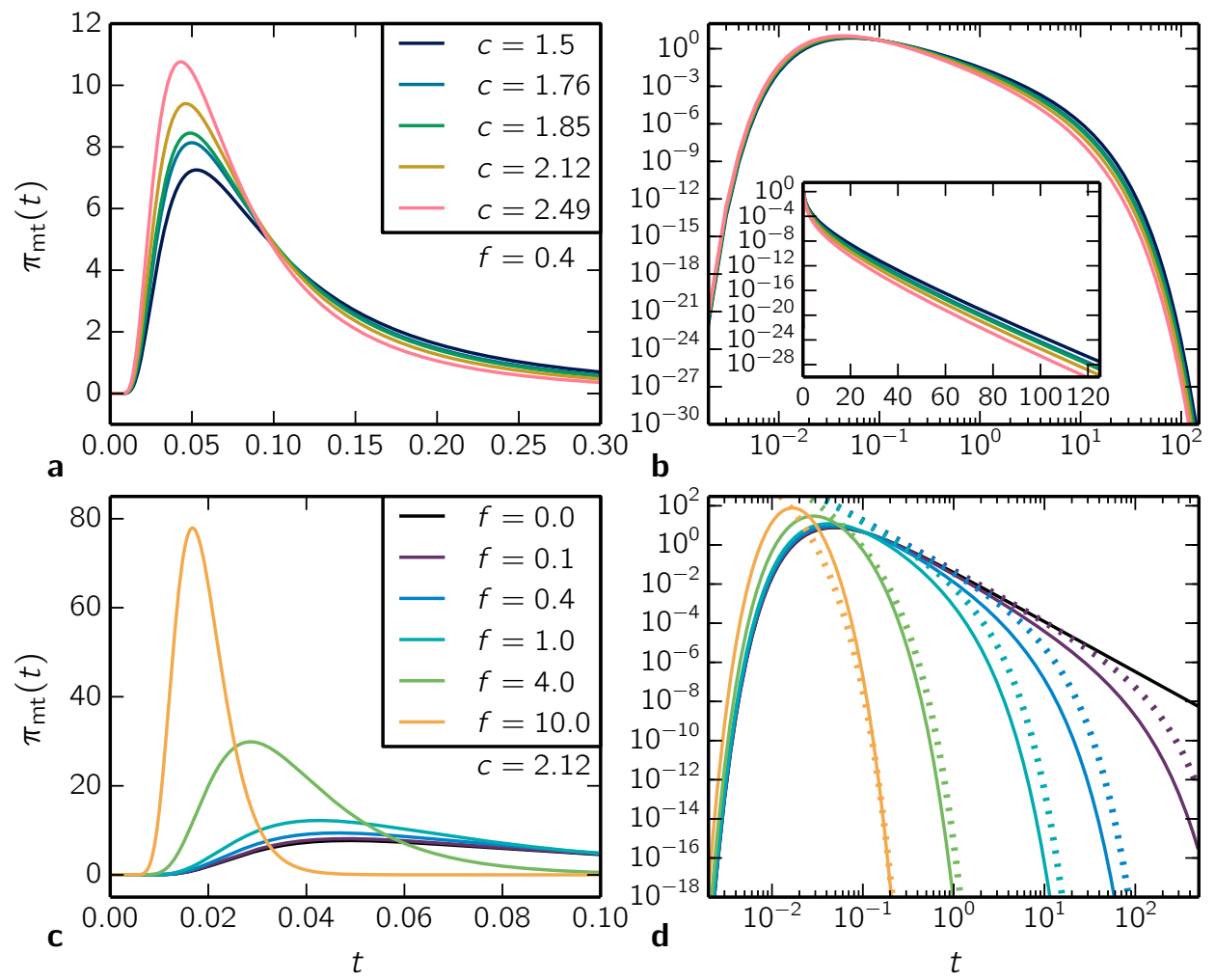

Figure 3. Meeting time PDFs. a,b) Dependence of the meeting time PDFs $\pi_{\mathrm{mt}}(t)$ on $c$ for $f=0.4$ and $x_{0}=1$. c,d) Dependence of the meeting time PDFs $\pi_{\mathrm{mt}}(t)$ on $f$ for $c=2.12$ and $x_{0}=1$. The asymptotic behaviour exhibits a transition from power law controlled by $c$ to an exponential decay given by $f^{2}$, except for $T=T_{\mathrm{c}}$ (corresponding to $f=0$ ), where the power law holds for all times. Dotted lines are the asymptotic functions (3.12) from Ref. 9].

where $y_{0}$ is the initial centre position. Without loss of generality, we set $y_{0}=0$. The bubble position PDF is

$$
P_{\mathrm{c}}(y, t \mid 0)=\frac{1}{\sqrt{2 \pi t}} \exp \left(-\frac{y^{2}}{2 t}\right) .
$$

Integrating the product of the meeting time PDF and the bubble position PDF over all times yields the meeting position PDF

$$
\pi_{\mathrm{mp}}\left(y \mid x_{0}, y_{0}\right)=\int_{0}^{\infty} \mathrm{d} t \pi_{\mathrm{mt}}\left(t \mid x_{0}\right) P_{\mathrm{c}}\left(y, t \mid y_{0}\right) .
$$

The time when the bubble edges meet is projected onto the meeting position which leads to the equivalence of the Fourier transform of the meeting position PDF and the Laplace transform of the meeting time PDF. We refer to this equivalence as the projection principle because closing dynamics of the bubble in the time variable $t$ are completely projected onto the closing dynamics in the spatial variable $y$. 
The Fourier transform of the definition of the meeting position distribution (3.15) (denoted by tilde) reads

$$
\mathcal{F}\left[\pi_{\mathrm{mp}}\right]\left(k \mid x_{0}\right) \equiv \tilde{\pi}_{\mathrm{mp}}\left(k \mid x_{0}\right)=\int_{0}^{\infty} \mathrm{d} t \pi_{a b s}\left(t \mid x_{0}\right) \tilde{P}_{c}(k, t),
$$

where we use the unitary Fourier transform $\mathcal{F}[h(y)](k)=(2 \pi)^{-1 / 2} \int_{-\infty}^{\infty} h(y) \exp (-i k y) \mathrm{d} y$. The Fourier transform of the probability distribution function of the position of the bubble-centre PDF is given by

$$
\tilde{P}_{c}(k, t)=\frac{1}{\sqrt{2 \pi}} e^{-\frac{k^{2}}{2} t} .
$$

Inserting this expression into (3.16) yields

$$
\tilde{\pi}_{\mathrm{mp}}\left(k \mid x_{0}\right)=\frac{1}{\sqrt{2 \pi}} \int_{0}^{\infty} \mathrm{d} t \pi_{\mathrm{mt}}\left(t \mid x_{0}\right) e^{-\frac{k^{2}}{2} t} .
$$

Finally, we compare the previous relation with the Laplace transform of the meeting time probability density

$$
\mathcal{L}\left[\pi_{\mathrm{mt}}\left(t \mid x_{0}\right)\right]\left(s \mid x_{0}\right) \equiv \bar{\pi}_{\mathrm{mt}}\left(s \mid x_{0}\right)=\int_{0}^{\infty} \mathrm{d} t \pi_{\mathrm{mt}}\left(t \mid x_{0}\right) e^{-s t}
$$

which leads to the relation

$$
\tilde{\pi}_{\mathrm{mp}}\left(k \mid x_{0}\right)=\left.\frac{1}{\sqrt{2 \pi}} \bar{\pi}_{\mathrm{mt}}\left(s \mid x_{0}\right)\right|_{s \rightarrow \frac{k^{2}}{2}} .
$$

This is the explicit form of the projection principle described above.

The Fourier picture expression for the meeting position PDF is

$$
\begin{aligned}
\tilde{\pi}_{\mathrm{mp}}\left(k \mid x_{0}\right)= & \frac{1}{\sqrt{2 \pi}} \frac{e^{f x_{0}}}{\Gamma(1+2 \gamma)}\left(x_{0} \sqrt{4 f^{2}+k^{2}}\right)^{\gamma} \Gamma\left(1+\gamma+\frac{2 f \gamma}{\sqrt{4 f^{2}+k^{2}}}\right) \times \\
& \times W\left(-\frac{2 f \gamma}{\sqrt{4 f^{2}+k^{2}}} ; \gamma+\frac{1}{2}\right)\left(x_{0} \sqrt{4 f^{2}+k^{2}}\right) .
\end{aligned}
$$

Its inverse Fourier transform can be obtained numerically using Fast Fourier transform (FFT) based algorithms [41, Sec. 13.8]. For $f=0$, the result can be expressed analytically in the form of a modified Lorenz distribution

$$
\pi_{\mathrm{mp}}\left(y \mid x_{0}, y_{0}\right)=\frac{2 x_{0}^{1+c}}{\sqrt{\pi}} \frac{\Gamma\left(\frac{c}{2}+1\right)}{\Gamma\left(\frac{c}{2}+\frac{1}{2}\right)}\left(x_{0}^{2}+4\left(y-y_{0}\right)^{2}\right)^{-\left(1+\frac{c}{2}\right)} .
$$

For non-zero $f$, we again observe an exponential suppression of the PDF tails (Figure 4).

\section{Subdiffusive dynamics}

\subsection{Fractional Fokker-Planck equation}

Subdiffusion can be described similarly to diffusion, by introducing fractional time derivatives. The fractional Fokker-Planck equation (FFPE) is the subdiffusive analogue 

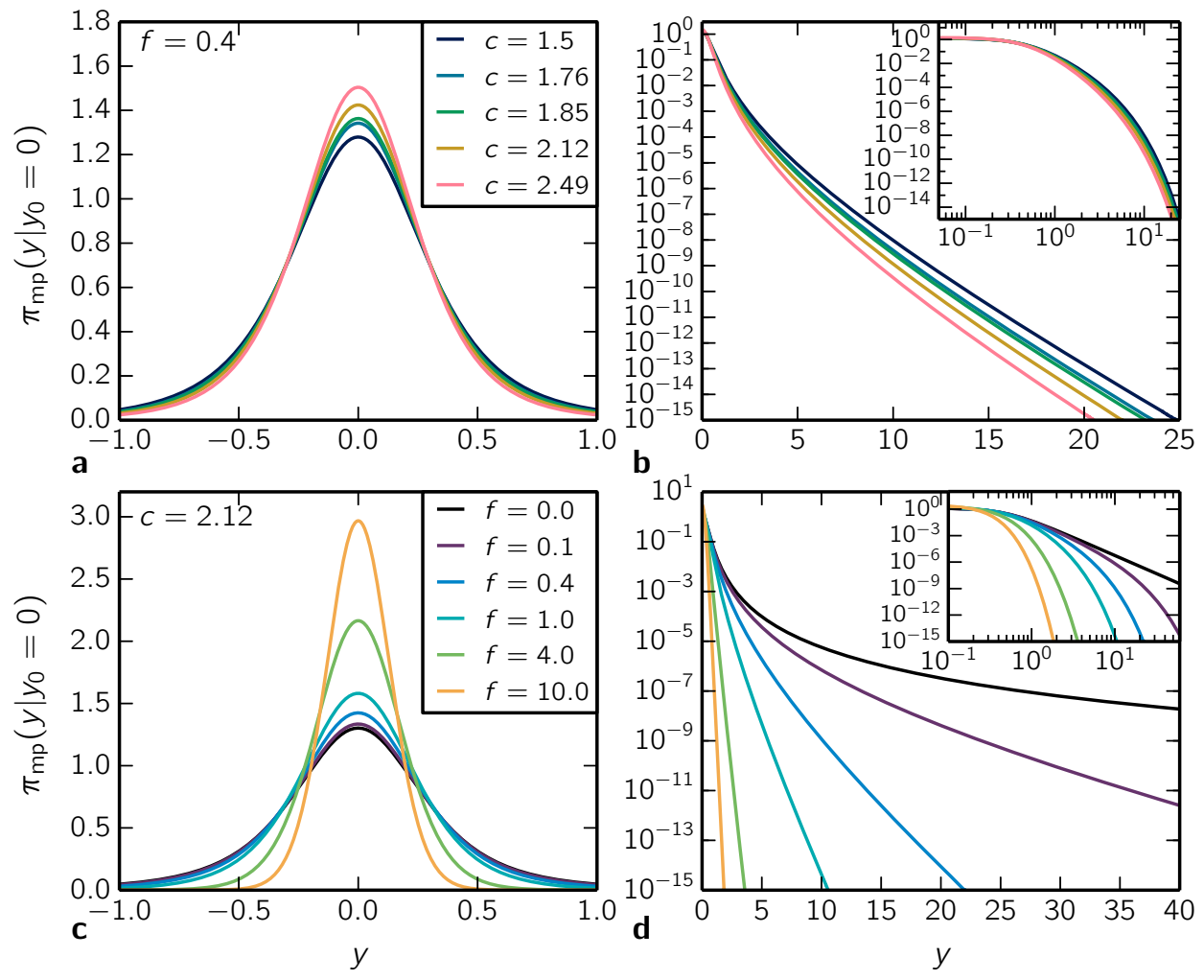

Figure 4. Meeting position PDFs. a,b) Dependence of the meeting position PDFs on $c$ for $f=0.4$ and $x_{0}=1, y_{0}=0$. $\left.\mathbf{c}, \mathbf{d}\right)$ Dependence of the meeting position PDFs on $f$ for $c=2.12$ and $x_{0}=1, y_{0}=0$. The asymptotics show a transition from the power law behaviour at the melting temperature $(f=0)$ to an exponential decay for finite $f$. This is similar to the case of the meeting time PDF, although the onset of the exponential behaviour is more pronounced for the meeting position.

of the diffusive FPE (3.1). Introduction to and mathematical background of subdiffusion and FFPE is given in reviews [42, 43]. The bubble-size FFPE reads

$$
\begin{aligned}
\partial_{\tau} P_{\mathrm{s}}^{(\nu)}\left(x, \tau \mid x_{0}\right) & =-\partial_{x} S_{\mathrm{s}}^{(\nu)}\left(x, \tau \mid x_{0}\right) \\
& ={ }_{0} \mathcal{D}_{\tau}^{1-\nu} K_{\nu} \partial_{x}\left[\partial_{x} P_{\mathrm{s}}^{(\nu)}\left(x, \tau \mid x_{0}\right)+\beta P_{\mathrm{s}}^{(\nu)}\left(x, \tau \mid x_{0}\right) \partial_{x} G(x)\right],
\end{aligned}
$$

where ${ }_{0} \mathcal{D}_{\tau}^{1-\nu}$ is the Riemann-Liouville differential operator [42]

$$
{ }_{0} \mathcal{D}_{\tau}^{1-\nu} f(\tau) \equiv \frac{\partial_{\tau}}{\Gamma(\nu)} \int_{0}^{\tau} \mathrm{d} \tau^{\prime} \frac{f\left(\tau^{\prime}\right)}{\left(\tau-\tau^{\prime}\right)^{1-\nu}} .
$$

Solutions of FFPE are subordinate to solutions of the corresponding FPE 43

$$
\bar{P}_{\mathrm{s}}^{(\nu)}\left(x, s \mid x_{0}\right)=s^{\nu-1} \bar{P}_{\mathrm{s}}\left(x, s^{\nu} \mid x_{0}\right) .
$$

A similar relation exists for the current. Comparing (3.1) and (4.1) gives

$$
\bar{S}_{\mathrm{s}}^{(\nu)}\left(x, s \mid x_{0}\right)=\bar{S}_{\mathrm{s}}\left(x, s^{\nu} \mid x_{0}\right) .
$$




\subsection{Subdiffusive Green function}

Since the transform (3.4) only acts on the spatial part of the bubble-size FPE, analogy of (3.5) can be found for subdiffusion, with dimensionless time $t=\tau\left[K_{\nu} / 2\right]^{1 / \nu}$

$$
\begin{gathered}
-\partial_{t} w^{(\nu)}\left(x, t \mid x_{0}\right)+{ }_{0} \mathcal{D}_{t}^{1-\nu}\left[2 \partial_{x x}-2 f^{2}-\frac{4 f \gamma}{x}-\frac{2 \gamma(\gamma+1)}{x^{2}}\right] w^{(\nu)}\left(x, t \mid x_{0}\right)=0 \\
w^{(\nu)}\left(x, t \rightarrow 0^{+} \mid x_{0}\right)=\delta\left(x-x_{0}\right), \quad w^{(\nu)}\left(x \rightarrow 0^{+}, t \mid x_{0}\right)=0 .
\end{gathered}
$$

Using (4.3), we find the corresponding subdiffusive Green function

$$
\begin{aligned}
\bar{P}_{\mathrm{s}}^{(\nu)}\left(x, s \mid x_{0}\right)= & s^{\nu-1} e^{-f\left(x-x_{0}\right)}\left(\frac{x}{x_{0}}\right)^{-\gamma} \frac{\Gamma\left(1+\gamma+\frac{f \gamma}{\alpha_{\nu}(s)}\right)}{4 \alpha_{\nu}(s) \Gamma(2+2 \gamma)} \times \\
& \times\left[\theta\left(x-x_{0}\right) W_{\left(-\frac{f \gamma}{\alpha_{\nu}(s)} ; \gamma+\frac{1}{2}\right)}\left(2 \alpha_{\nu}(s) x\right) M_{\left(-\frac{f \gamma}{\alpha_{\nu}(s)} ; \gamma+\frac{1}{2}\right)}\left(2 \alpha_{\nu}(s) x_{0}\right)+\right. \\
& \left.+\theta\left(x_{0}-x\right) M_{\left(-\frac{f \gamma}{\alpha_{\nu}(s)} ; \gamma+\frac{1}{2}\right)}\left(2 \alpha_{\nu}(s) x\right) W_{\left(-\frac{f \gamma}{\alpha_{\nu}(s)} ; \gamma+\frac{1}{2}\right)}\left(2 \alpha_{\nu}(s) x_{0}\right)\right], \\
& \text { where } \quad \alpha_{\nu}(s)=\sqrt{f^{2}+s^{\nu} / 2} .
\end{aligned}
$$

\subsection{Meeting time PDF}

The subdiffusive meeting time PDF follows from substituting (3.11) into (4.4) and has again the meaning of the probability flow into the boundary due to the bubble closing events

$$
\begin{aligned}
\bar{\pi}_{\mathrm{mt}}^{(\nu)}\left(s \mid x_{0}\right)= & \frac{e^{f x_{0}}}{\Gamma(1+2 \gamma)}\left(x_{0} \sqrt{4 f^{2}+2 s^{\nu}}\right)^{\gamma} \Gamma\left(1+\gamma+f \gamma \sqrt{\frac{2}{2 f^{2}+s^{\nu}}}\right) \times \\
& \times W_{\left(-f \gamma \sqrt{\frac{2}{2 f^{2}+s^{\nu}}} ; \gamma+\frac{1}{2}\right)}\left(x_{0} \sqrt{4 f^{2}+2 s^{\nu}}\right) .
\end{aligned}
$$

The inverse Laplace transform is obtained numerically as in the diffusive case (Section 3) and plotted in Figure 5. We observe a universal behaviour for large times and all values of $f$ and $c$ studied, where the meeting time PDFs decay as a power law with exponent $-(1+\nu)$ (see Figure 5).

We propose the following explanation of this effect. We assume the existence of a finite mean meeting time

$$
T_{1}=-\lim _{s \rightarrow 0^{+}} \frac{\bar{\pi}_{\mathrm{mt}}\left(s \mid x_{0}\right)}{s} .
$$

of the diffusive meeting time PDF. Due to $\pi_{\mathrm{mt}}$ being bounded and having asymptotics (3.12), the finiteness of $T_{1}$ is guaranteed for all $c>0$ below the melting temperature $(f>0)$ and for $c>1$ at the transition $(f=0)$. The meeting time PDF can be expanded around $s=0$ as $\bar{\pi}_{\mathrm{mt}}\left(s \mid x_{0}\right)=1-T_{1} s+\mathcal{O}\left(s^{2}\right)$ in the Laplace picture. Subordination gives $\bar{\pi}_{\mathrm{mt}}^{(\nu)}\left(s \mid x_{0}\right)=1-T_{1} s^{\nu}+\mathcal{O}\left(s^{2 \nu}\right)$ for small $s$ in the subdiffusive case. Tauberian theorems [44, Ch. XIII, Eq. (5.22)] lead to $\pi_{\mathrm{mt}}^{(\nu)}\left(t \rightarrow \infty \mid x_{0}\right)=t^{-(1+\nu)}+\mathcal{O}\left(t^{-(1+2 \nu)}\right)$ in the time domain for $\nu<1$. Alternatively, the scaling can be derived by considering the subdiffusive dynamics to be a limit of a continuous time random walk [45, 46, 47]. 

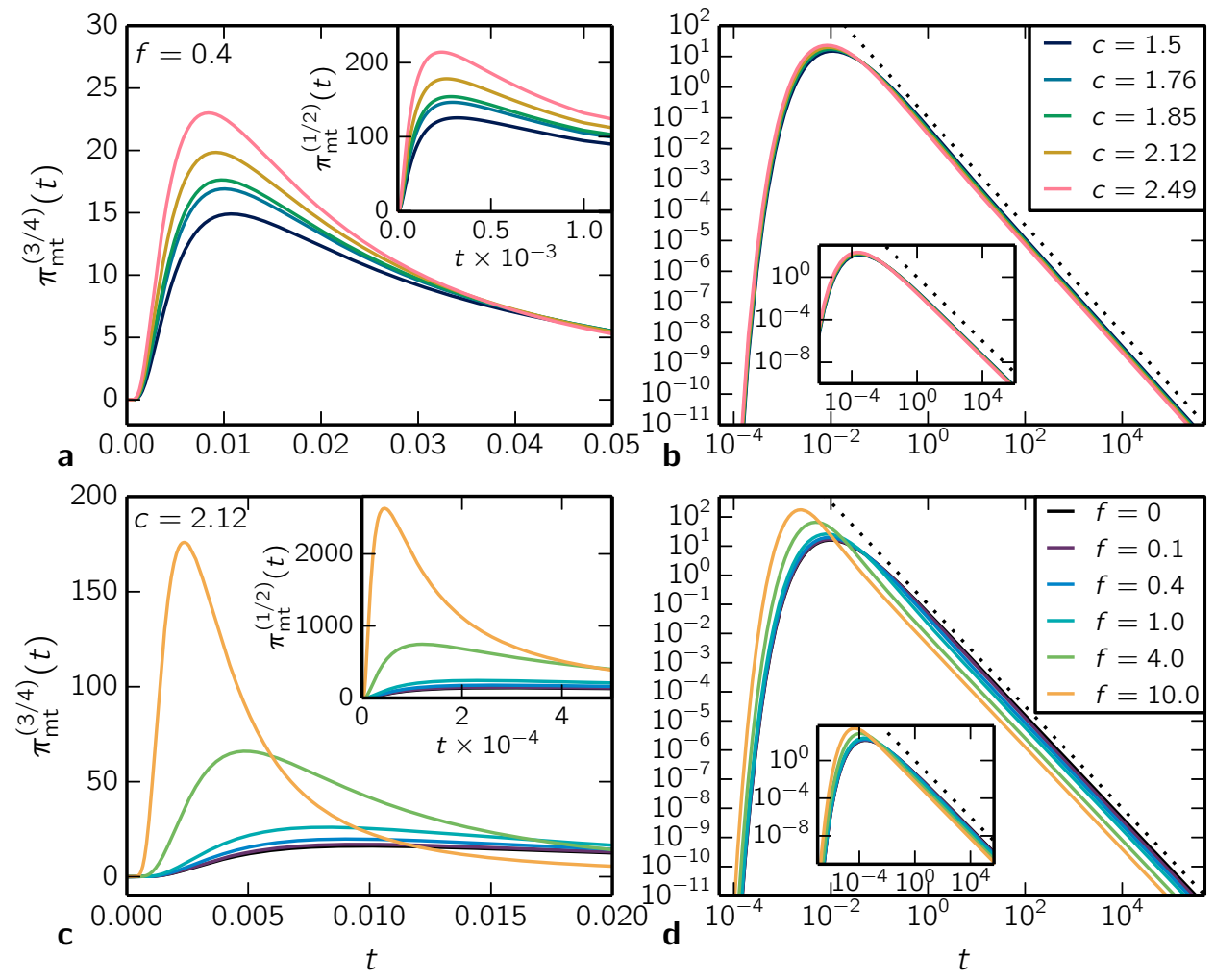

Figure 5. Meeting time PDFs for subdiffusion. a,b) Dependence of the meeting time PDFs on $c$ for $f=0.4$ and $x_{0}=1$. $\left.\mathbf{c}, \mathbf{d}\right)$ Dependence of the meeting time PDFs on $f$ for $c=2.12$ and $x_{0}=1$. The main figures show results for $\nu=3 / 4$ while the insets those for $\nu=1 / 2$. The dotted lines show the universal power-law behaviour with the exponent $-(1+\nu)$.

In contrast to the universal behaviour of the tails, peaks of meeting time PDFs show a very strong dependence on $f$ and $c$, which is even more pronounced than in the diffusive case. The peak values of the subdiffusive meeting time PDF surpass those in the diffusive case. Returning to the physical origin of the subdiffusive behaviour allows us to explain this behaviour.

The subdiffusive regime appears as the heterogeneity of the DNA sequence leads to the trapping of bubbles in AT-rich (soft) regions surrounded by GC-rich regions [24]. The hard-to-open GC-rich regions limit the growth of the bubble, which can be likened to the presence of a reflective boundary condition. The presence of a boundary decreases the mean meeting time, as we observe below in the case of finite DNA chains. However, if the bubble overcomes such a GC-rich barrier, it spreads out to the next AT-rich region and its survival time increases. This corresponds to the power law behaviour in tails which results in a small fraction of long-lived bubbles which is larger than in the diffusive case. 


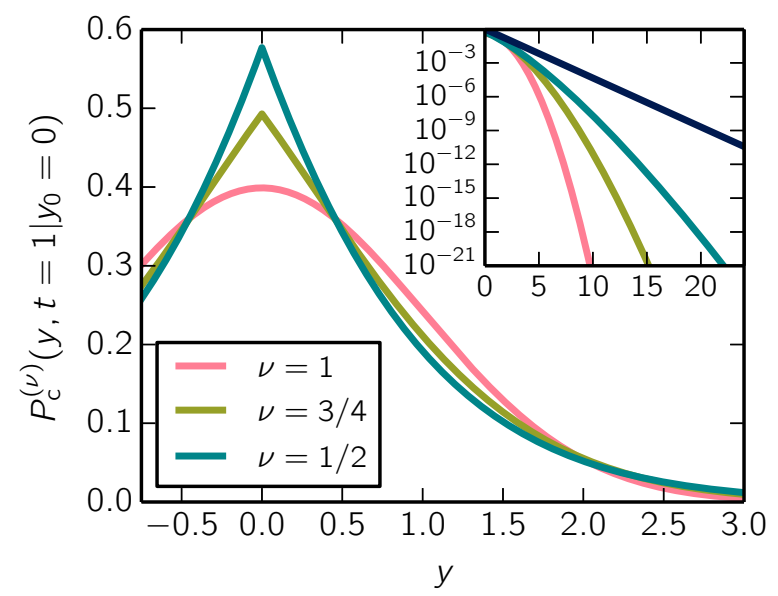

Figure 6. Bubble centre PDFs for free subdiffusion. $P_{\mathrm{c}}^{(\nu)}\left(y, t=1 \mid y_{0}=0\right)$ given by Eq. (4.10) for selected values of $\nu(\nu=1$ corresponding to the standard diffusion (3.14) is shown for comparison). The cusp at $y=0$ for $\nu<1$ is a characteristic of the subdiffusive behaviour. Inset: large-distance asymptotics are given by exponentials of powers (bounded between 1 and 2, compare with Eqs. (4.12) and (4.13) ) of $y$. Dark blue topmost straight line shows a simple exponential for comparison.

\subsection{Meeting position PDF}

The definition of the meeting position PDF (3.15) holds for subdiffusive dynamics as well. However, subdiffusive dynamics do not lead to a simple Gaussian form of the bubble position PDF and, therefore, the projection principle cannot be used. Subdiffusive bubble position PDF solves the appropriately modified Eq. (3.13)

$$
\partial_{t} P_{\mathrm{c}}^{(\nu)}\left(y, t \mid y_{0}\right)={ }_{0} \mathcal{D}_{t}^{1-\nu} \partial_{y y} P_{\mathrm{c}}^{(\nu)}\left(y, t \mid y_{0}\right),
$$

with the following scaling property of the solution $P_{\mathrm{c}}^{(\nu)}\left(y, t \mid y_{0}\right)=P_{\mathrm{c}}^{(\nu)}((y-$ $\left.\left.y_{0}\right) / t^{\nu / 2}, 1 \mid 0\right) / t^{\nu / 2}$ stemming directly from the joint Laplace and Fourier transform of Eq. (4.9). Explicitly, the solution is described in terms of the Fox H-functions [42]

$$
P_{\mathrm{c}}^{(\nu)}\left(y, t \mid y_{0}\right)=\frac{1}{\sqrt{2 \pi t^{\nu}}} H_{1,2}^{2,0}\left[\frac{\left(y-y_{0}\right)^{2}}{2 t^{\nu}} \mid \begin{array}{l}
\left(1-\frac{\nu}{2}, \nu\right) \\
(0,1),\left(\frac{1}{2}, 1\right)
\end{array}\right],
$$

which reduce to the Meijer G-functions (more commonly implemented in numerical libraries than the Fox H-functions), if $\nu=q / p$ is rational. However, the number of Meijer G-function parameters increases with growing $q$ and $p$, increasing the computational effort for evaluating the functions and decreasing their precision. Useful special cases are $\nu=1 / 2$ and $\nu=3 / 4$, for which the bubble centre PDFs take on the forms

$$
\begin{aligned}
& P_{\mathrm{c}}^{(1 / 2)}\left(y, t \mid y_{0}\right)=\frac{1}{2 \pi^{\frac{3}{2}} t^{\frac{1}{4}}} G_{0,3}^{3,0}\left[\frac{\left(y-y_{0}\right)^{4}}{64 t} \mid \overline{\left(0, \frac{1}{4}, \frac{1}{2}\right)}\right], \\
& P_{\mathrm{c}}^{(3 / 4)}\left(y, t \mid y_{0}\right)=\frac{1}{2 \sqrt{2} 3^{\frac{1}{8}} \pi^{\frac{5}{2}} t^{\frac{3}{8}}} G_{2,7}^{7,0}\left[\frac{\left(y-y_{0}\right)^{8}}{32^{4} t^{3}} \mid \begin{array}{l}
\left(\frac{5}{24}, \frac{13}{24}\right) \\
\left(0, \frac{1}{8}, \frac{1}{4}, \frac{3}{8}, \frac{1}{2}, \frac{5}{8}, \frac{3}{4}\right)
\end{array}\right],
\end{aligned}
$$


with asymptotics reading

$$
\begin{aligned}
& P_{\mathrm{c}}^{(1 / 2)}(u \rightarrow \infty, 1 \mid 0) \propto \exp \left(-\frac{3}{4} u^{\frac{4}{3}}\right)\left(u^{-\frac{1}{3}}+\mathcal{O}\left(u^{-\frac{4}{3}}\right)\right), \\
& P_{\mathrm{c}}^{(3 / 4)}(u \rightarrow \infty, 1 \mid 0) \propto \exp \left(-\frac{3^{\frac{3}{5}} 5}{16} u^{\frac{8}{5}}\right)\left(u^{-\frac{1}{5}}+\mathcal{O}\left(u^{-\frac{6}{5}}\right)\right) .
\end{aligned}
$$

These functions, along with the Gaussian (3.14) for comparison, are plotted in Figure 6] which shows their characteristic non-analytic behaviour at zero (cusp, i.e. the discontinuity in first derivative). The inset shows their localisation around zero (stronger than exponential but weaker than Gaussian).
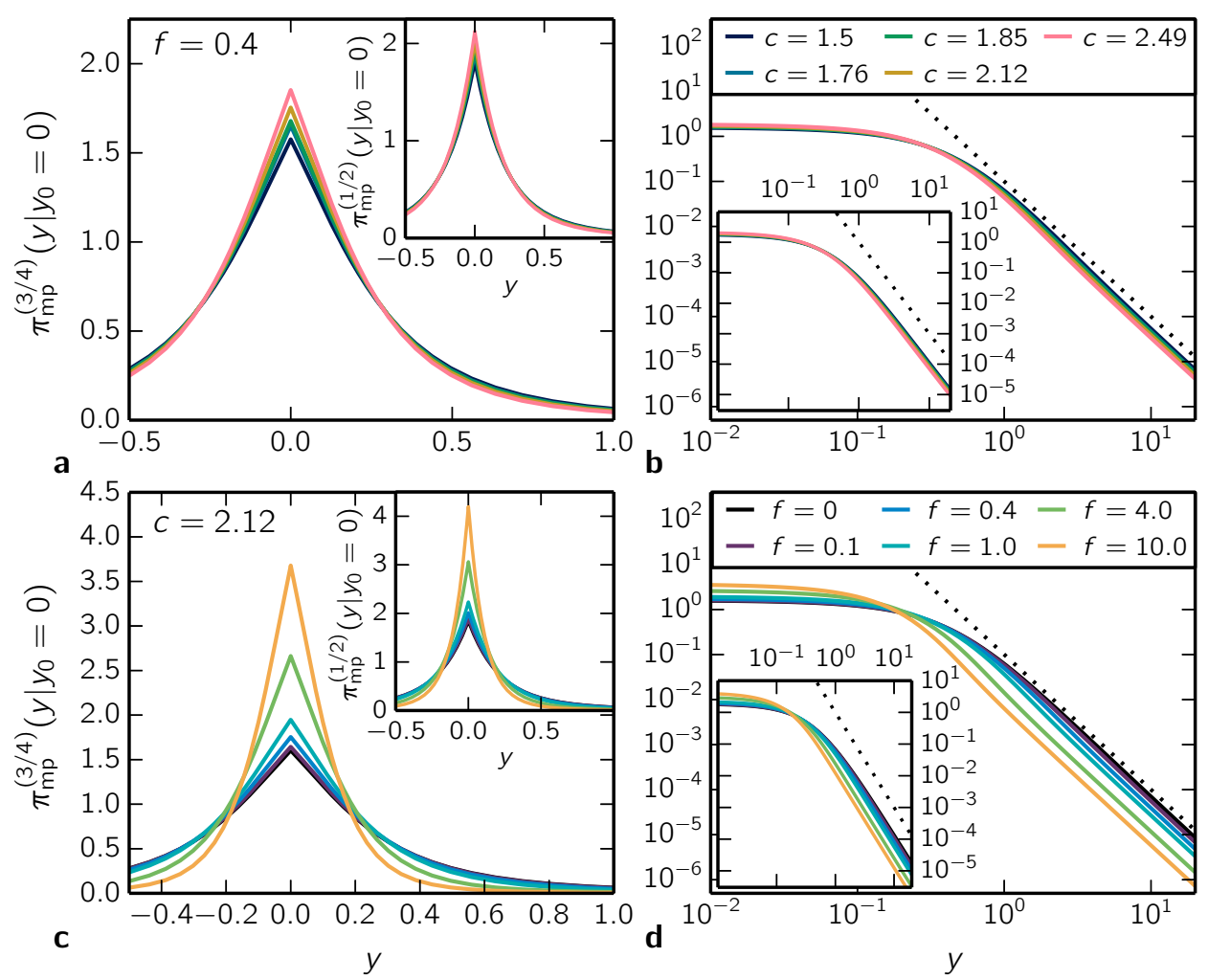

Figure 7. Meeting position PDFs for subdiffusion $\left(y_{0}=0, x_{0}=1\right)$. a,b) Dependence of the meeting position PDFs on $c$ for $f=0.4$. $\mathbf{c}, \mathbf{d})$ Dependence of the meeting position PDFs on $f$ for $c=2.12$. The main figures show results for $\nu=3 / 4$ while the insets those for $\nu=1 / 2$. The cusp from the bubble centre PDFs (see Figure6) is projected into the meeting position PDFs. The peak value increases with decreasing $\nu$ (also compare with Figure 4). The PDFs exhibit universal power law asymptotics $y^{-3}$, which are independent of $\nu, c$ and $f$ - dotted lines.

These localisation properties can be used in the calculation and analysis of the meeting position PDFs determined by the integral (3.15)

$\pi_{\mathrm{mp}}^{(\nu)}\left(y \mid x_{0}\right)=\int_{0}^{\infty} \mathrm{d} t \pi_{\mathrm{mt}}^{(\nu)}\left(t \mid x_{0}\right) P_{\mathrm{c}}^{(\nu)}(y, t \mid 0)=\int_{0}^{\infty} \frac{\mathrm{d} t}{t^{\frac{\nu}{2}}} \pi_{\mathrm{mt}}^{(\nu)}\left(t \mid x_{0}\right) P_{c}^{(\nu)}\left(\frac{y}{t^{\frac{\nu}{2}}}, 1 \mid 0\right)$. 
Now, assuming $y>0$ (both $P_{c}^{(\nu)}(y, t \mid 0)$ and, consequently, also $\pi_{\mathrm{mp}}^{(\nu)}\left(y \mid x_{0}\right)$ are even functions of $y$ ), we substitute $t \rightarrow \eta=y / t^{\nu / 2}$ in the above integral, which yields

$$
\pi_{\mathrm{mp}}^{(\nu)}\left(y \mid x_{0}\right)=\frac{2}{\nu} y^{\frac{2}{\nu}-1} \int_{0}^{\infty} \mathrm{d} \eta \eta^{-\frac{2}{\nu}} P_{\mathrm{c}}^{(\nu)}(\eta, 1 \mid 0) \pi_{\mathrm{mt}}^{(\nu)}\left(\left(\frac{y}{\eta}\right)^{\frac{2}{\nu}} \mid x_{0}\right) .
$$

This equation was used for the numerical evaluation of the meeting position PDFs $\pi_{\mathrm{mp}}^{(\nu)}\left(y \mid x_{0}\right)$ depicted in Figure 7. Furthermore, the super-exponential localisation of the integrand factor $\eta^{-2 / \nu} P_{\mathrm{c}}^{(\nu)}(\eta, 1 \mid 0)$ in Eq. (4.15) around zero together with the powerlaw decay of $\pi_{\mathrm{mt}}^{(\nu)}\left(t \rightarrow \infty \mid x_{0}\right) \approx t^{-(1+\nu)}$ derived in Sec. 4.3 give a surprisingly universal expression for the large-distance asymptotics

$$
\pi_{\mathrm{mp}}^{(\nu)}\left(y \rightarrow \infty \mid x_{0}\right) \approx y^{-3} \times \frac{2}{\nu} \int_{0}^{\infty} d \eta \eta^{2} P_{c}^{(\nu)}(\eta, 1 \mid 0),
$$

whose power exponent is just -3 , completely independent of any parameters of the model such as $f, c$, and, most remarkably, also $\nu$. This universality is demonstrated in Figure 7 for the two cases with $\nu=1 / 2$ and $\nu=3 / 4$.

\section{Dynamics in finite DNA chains}

In a finite DNA molecule, the diffusive dynamics of bubble position and its size depend on each other because the boundary conditions break the translational invariance of the infinite-length problem and, thus, connect the two variables. We need to consider a joint $\operatorname{PDF} P_{\mathrm{e}}\left(\xi, \chi, t \mid \xi_{0}, \chi_{0}\right)$ of the edge positions $\xi, \chi$ that do not cross: $\xi<\chi$. We rescale $\xi$ and $\chi$ to take values between 0 and 1 (see the discussion on scaling below). The bubble size $x$ and bubble position $y$ relate to the edge locations through $\xi=y-x / 2$ and $\chi=y+x / 2$.

\subsection{Two-dimensional Fokker Planck equation}

Keeping the same form of the Gibbs free energy as in the infinite chain case (2.1), the two-dimensional FPE reads

$$
\begin{gathered}
{\left[-\partial_{t}+\partial_{\xi \xi}+\partial_{\chi \chi}+\left(\partial_{\chi}-\partial_{\xi}\right)\left(2 f+\frac{2 \gamma}{\chi-\xi}\right)\right] P_{\mathrm{e}}\left(\xi, \chi, t \mid \xi_{0}, \chi_{0}\right)=0,} \\
P_{\mathrm{e}}\left(\xi, \chi, t \rightarrow 0^{+} \mid \xi_{0}, \chi_{0}\right)=\delta\left(\xi-\xi_{0}\right) \delta\left(\chi-\chi_{0}\right) .
\end{gathered}
$$

We track the closing dynamics of a single loop and impose the absorbing boundary condition

$$
\lim _{\chi \rightarrow \xi^{+}} P_{\mathrm{e}}\left(\xi, \chi, t \mid \xi_{0}, \chi_{0}\right)=0 .
$$

Bubble edges behave as vicious walkers [48, 49] that annihilate each other upon meeting. This picture has already helped investigating coalescence of DNA bubbles in Refs. [26, 27]. 
In our model, the single strands of DNA are considered to be clamped together at the ends of the molecule. This assumption leads to the reflecting boundary conditions

$$
\begin{aligned}
& {\left.\left[\partial_{\xi}-2 f-\frac{2 \gamma}{\chi-\xi}\right] P_{\mathrm{e}}\left(\xi, \chi, t \mid \xi_{0}, \chi_{0}\right)\right|_{\xi=0}=0} \\
& {\left.\left[\partial_{\chi}+2 f+\frac{2 \gamma}{\chi-\xi}\right] P_{\mathrm{e}}\left(\xi, \chi, t \mid \xi_{0}, \chi_{0}\right)\right|_{\chi=1}=0 .}
\end{aligned}
$$

Admissible positions of the edges form an isosceles right-angled triangle with the absorbing condition on the hypotenuse and the reflecting conditions on the legs, described as $\Omega$ in Figure 8 ,

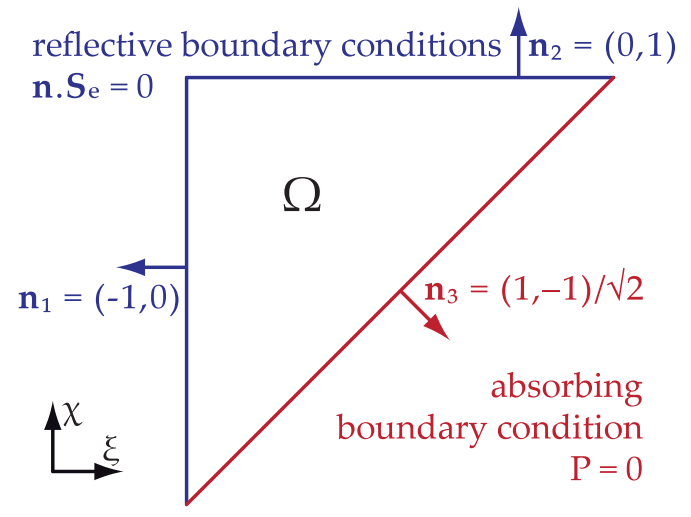

Figure 8. Support $\Omega$ and boundary conditions of the finite-chain bivariate Fokker-Planck partial differential equation (5.1). The reflective boundary conditions (blue legs) correspond to the ends of the DNA chain where the strands are clamped together. The absorbing boundary condition (red hypotenuse) corresponds to closing of the bubble if $\xi=\chi$.

The effect of the entropic term $\gamma / x$ relative to the energetic term $f$ is strongest for small bubbles or for low $f$, i.e., close to the melting temperature. The equations (3.1), (4.1), and (5.1) can be rescaled as follows

$$
\begin{aligned}
& x=A x^{\prime} \\
& f=f^{\prime} / A \\
& \gamma=\gamma^{\prime} \\
& t=A^{2 / \nu} t^{\prime} .
\end{aligned}
$$

Note that the loop exponent $c=2 \gamma$ stays unchanged under the scaling. Position variables $y, \xi$, and $\chi$ transform in the same fashion as $x$. The principal relation is $f x=f^{\prime} x^{\prime}$, because the scaling of the time variable can be absorbed into the fitting of the diffusion constant. The measure $P_{\mathrm{e}}\left(\xi, \chi, t \mid \xi_{0}, \chi_{0}\right) \mathrm{d} \xi \mathrm{d} \chi$ stays invariant in order to ensure a consistent norming of probability, yielding $P_{\mathrm{e}}\left(\xi, \chi, t \mid \xi_{0}, \chi_{0}\right)=A^{-2} P_{\mathrm{e}}\left(\xi^{\prime}, \chi^{\prime}, t^{\prime} \mid \xi_{0}^{\prime}, \chi_{0}^{\prime}\right)$, from which the scaling of the initial and boundary conditions follows (compare also with the discussion below Eq. (4.9) ). 


\subsection{Time-integrated quantities}

We solve the dynamics in finite chains numerically. Derived quantities - the first moment of the meeting time and the meeting position PDF - need only time-integrated information, which reduces the numerical effort.

5.2.1. Moments of the meeting time PDF. Closing of a bubble reduces the proportion of bubbles surviving; the meeting time PDF equals the negative of the time derivative of the spatial integral of $P_{\mathrm{e}}\left(\xi, \chi, t \mid \xi_{0}, \chi_{0}\right)$ over $\Omega$ [31, Sec. 8.1]

$$
\begin{aligned}
\pi_{\mathrm{mt}}\left(t \mid \xi_{0}, \chi_{0}\right) & =-\frac{\mathrm{d}}{\mathrm{d} t} \int_{\Omega} \mathrm{d} \xi \mathrm{d} \chi P_{\mathrm{e}}\left(\xi, \chi, t \mid \xi_{0}, \chi_{0}\right)= \\
& =-\int_{0}^{1} \mathrm{~d} \xi \int_{\xi}^{1} \mathrm{~d} \chi \frac{\mathrm{d} P_{\mathrm{e}}\left(\xi, \chi, t \mid \xi_{0}, \chi_{0}\right)}{\mathrm{d} t} .
\end{aligned}
$$

The first moment of the meeting time PDF is defined by

$T_{1}\left(\xi_{0}, \chi_{0}\right) \quad=\int_{0}^{\infty} t \pi_{\mathrm{mt}}\left(t \mid \xi_{0}, \chi_{0}\right) \mathrm{d} t=\int_{\Omega} p_{1}\left(\xi, \chi \mid \xi_{0}, \chi_{0}\right) \mathrm{d} \Sigma \quad$, where

$p_{1}\left(\xi, \chi \mid \xi_{0}, \chi_{0}\right)=-\int_{0}^{\infty} t \frac{\mathrm{d} P_{\mathrm{e}}\left(\xi, \chi, t \mid \xi_{0}, \chi_{0}\right)}{\mathrm{d} t} \mathrm{~d} t=\int_{0}^{\infty} P_{\mathrm{e}}\left(\xi, \chi, t \mid \xi_{0}, \chi_{0}\right) \mathrm{d} t$.

We apply $\mathbb{L}_{\mathrm{FP}}$ - the spatial part of $(5.1)$ - on both sides of the previous equation and use that $P_{\mathrm{e}}\left(\xi, \chi, t \mid \xi_{0}, \chi_{0}\right)$ solves the $\mathrm{FPE} \mathbb{L}_{\mathrm{FP}} P_{\mathrm{e}}\left(\xi, \chi, t \mid \xi_{0}, \chi_{0}\right)=\partial_{t} P_{\mathrm{e}}\left(\xi, \chi, t \mid \xi_{0}, \chi_{0}\right)$ with the $\delta$-function initial condition (5.1) [31, Sec. 8.1]

$$
\mathbb{L}_{\mathrm{FP}} p_{1}\left(\xi, \chi \mid \xi_{0}, \chi_{0}\right)=-\delta\left(\xi-\xi_{0}\right) \delta\left(\chi-\chi_{0}\right) .
$$

We have determined the auxiliary function $p_{1}\left(\xi, \chi \mid \xi_{0}, \chi_{0}\right)$ numerically using the finite element method (FEM), which has the advantage that $\delta$-distribution has a simple implementation in the weak formulation of the FPE. A hierarchy of auxiliary functions exists for higher moments of $\pi_{\mathrm{mt}}\left(t \mid \xi_{0}, \chi_{0}\right)$ [31, Sec. 8.1] and the approach using FEM can be suitably generalised.

5.2.2. Mean meeting time In Figure 9, we compare the numerical results for $T_{1}$ in the case of finite chains with the infinite chain mean meeting time $T_{1}(\infty)$ obtained from Eqs. (4.8) and (3.11). We rescaled the results to keep the initial size of the bubble fixed at $x_{0}=1$, whereas we vary the total chain length $L$, using the scaling relations (5.5). We observe a rapid exponential convergence with a characteristic convergence length $\ell \ll 1$ to the infinite chain value, leading to $\left|T_{1}(10) / T_{1}(\infty)-1\right|<\epsilon_{\mathrm{FEM}} \simeq 10^{-5}$, thus being indistinguishable for the FEM implementation used here. The sole exception is the critical point, where $T_{1}(\infty)-T_{1}(L) \propto L^{-1}$. This is consistent with the entropic term being scale-free as shown in Eq. (5.5). The exponential scaling can be recovered by initially placing the bubble in the vicinity of one of the chain ends. Generally, the presence of ends reduces the mean meeting time as it prevents the bubble from spreading out as is demonstrated in Figure 10. 

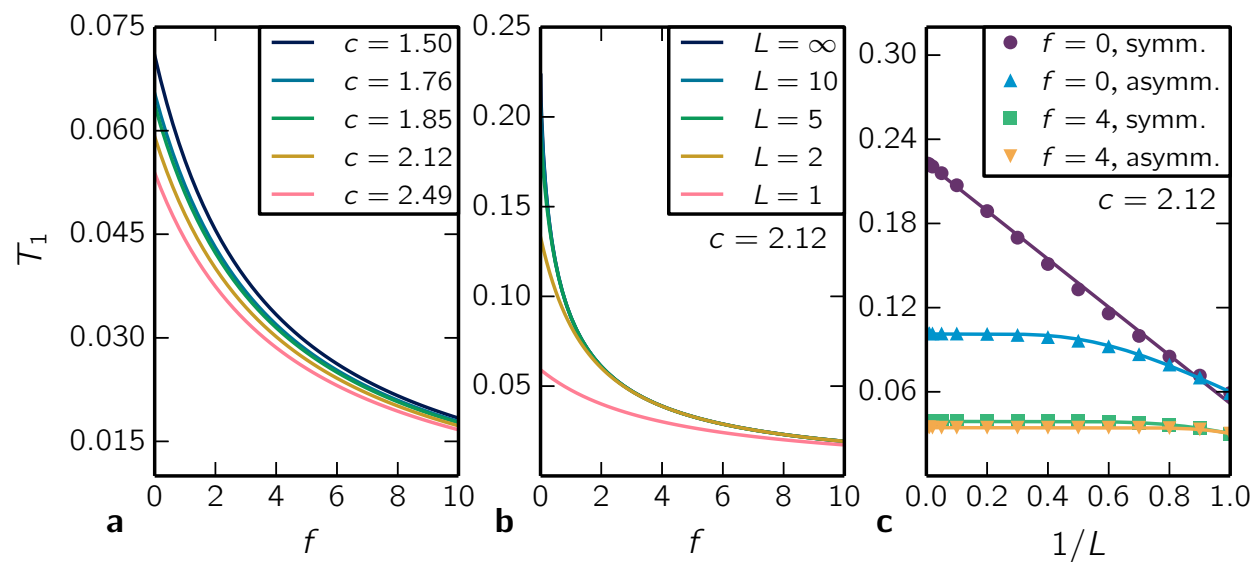

Figure 9. Mean meeting time - finite chain. a) Dependence of the mean meeting time $T_{1}$ on $c$ and $f$ for $L=1, \xi_{0}=0$, and $\chi_{0}=1$. b) Scaling of $T_{1}$ for a bubble of initial size $x_{0}=1$ in a chain of length $L$ in dependence on $f$. The infinite chain results are approached exponentially in case of finite $f$. c) Comparison of a bubble placed symmetrically in the centre of the chain with a bubble placed asymmetrically at its edge. Presence of the boundary causes the exponential scaling to remain at the melting temperature $(f=0)$, while the centrally placed bubble exhibits scaling linear with $L^{-1}$. Full lines are fits of linear $T_{1}(\infty)+\left(T_{1}(1)-T_{1}(\infty)\right) / L$ or exponential $T_{1}(\infty)+\left(T_{1}(1)-T_{1}(\infty)\right) \exp (-(L-1) / \ell)$ functions, respectively, with $T_{1}(\infty)$ being analytically known in the case of the symmetric initial condition or just another fitting parameter in the asymmetric case. The obtained values for the characteristic convergence length are $\ell(\mathbf{\Lambda})=0.42 \pm 0.02, \ell(\boldsymbol{\square})=0.192 \pm 0.001$, and $\ell(\mathbf{v})=0.096 \pm 0.006$.

5.2.3. Meeting position PDF The meeting position PDF is the time integrated probability that the bubble closes at a given position $\xi=\chi$ along the absorbing boundary. We restate it as the time-integrated projection of the probability current $\vec{S}_{\mathrm{e}}$ to the direction $\vec{n}_{3}$ orthogonally crossing the absorbing boundary in Fig. 8 taking on the following form

$$
\begin{aligned}
{\left[\vec{S}_{\mathrm{e}}\left(\xi, \chi, t \mid \xi_{0}, \chi_{0}\right) \cdot \overrightarrow{n_{3}}\right]_{\chi \rightarrow \xi^{+}} } & =\left[\left(-\partial_{\xi}+2 f+\frac{2 \gamma}{\chi-\xi},-\partial_{\chi}-2 f-\frac{2 \gamma}{\chi-\xi}\right) P_{\mathrm{e}}\left(\xi, \chi, t \mid \xi_{0}, \chi_{0}\right) \cdot \quad(5.10)\right. \\
& \left.\cdot \frac{1}{\sqrt{2}}(1,-1)\right]_{\chi \rightarrow \xi^{+}}=\frac{\sqrt{2}}{2}\left[\left(-\partial_{\xi}+\partial_{\chi}+\frac{4 \gamma}{\chi-\xi}\right) P_{\mathrm{e}}\left(\xi, \chi, t \mid \xi_{0}, \chi_{0}\right)\right]_{\chi \rightarrow \xi^{+}},
\end{aligned}
$$

where we have used the absorbing boundary condition to eliminate the term containing $f$. The time integral of the previous equation is

$$
\begin{aligned}
\pi_{\mathrm{mp}}\left(\xi \mid \xi_{0}, \chi_{0}\right) & =\int_{0}^{\infty} \mathrm{d} t\left[\vec{S}_{e}\left(\xi, \chi, t \mid \xi_{0}, \chi_{0}\right) \cdot \overrightarrow{n_{3}}\right]_{\chi \rightarrow \xi^{+}}= \\
& =\frac{1}{\sqrt{2}} \int_{0}^{\infty} \mathrm{d} t\left[\left(-\partial_{\xi}+\partial_{\chi}+\frac{4 \gamma}{\chi-\xi}\right) P_{e}\left(\xi, \chi, t \mid \xi_{0}, \chi_{0}\right)\right]_{\chi \rightarrow \xi^{+}}= \\
& =\frac{1}{\sqrt{2}}\left[\left(-\partial_{\xi}+\partial_{\chi}+\frac{4 \gamma}{\chi-\xi}\right) p_{1}\left(\xi, \chi \mid \xi_{0}, \chi_{0}\right)\right]_{\chi \rightarrow \xi^{+}} .
\end{aligned}
$$


Therefore the meeting position PDF can also be obtained from the first auxiliary function $p_{1}\left(\xi, \chi \mid \xi_{0}, \chi_{0}\right)$ as the first moment of the meeting time PDF above. Numerical observations show that both partial derivatives and $p_{1}\left(\xi, \chi \mid \xi_{0}, \chi_{0}\right) /(\chi-\xi)$ stay finite and non-zero as the limit $\chi \rightarrow \xi^{+}$is approached. Figure 10 shows results for selected parameter values. As in the case of the mean meeting time, we observe a rapid convergence of the meeting position PDF to the infinite chain values if the bubble is placed symmetrically. Placing the bubble at the extremity of the chain significantly modifies the shape of the PDF leading to a sharper peak around the initial position of the bubble centre.
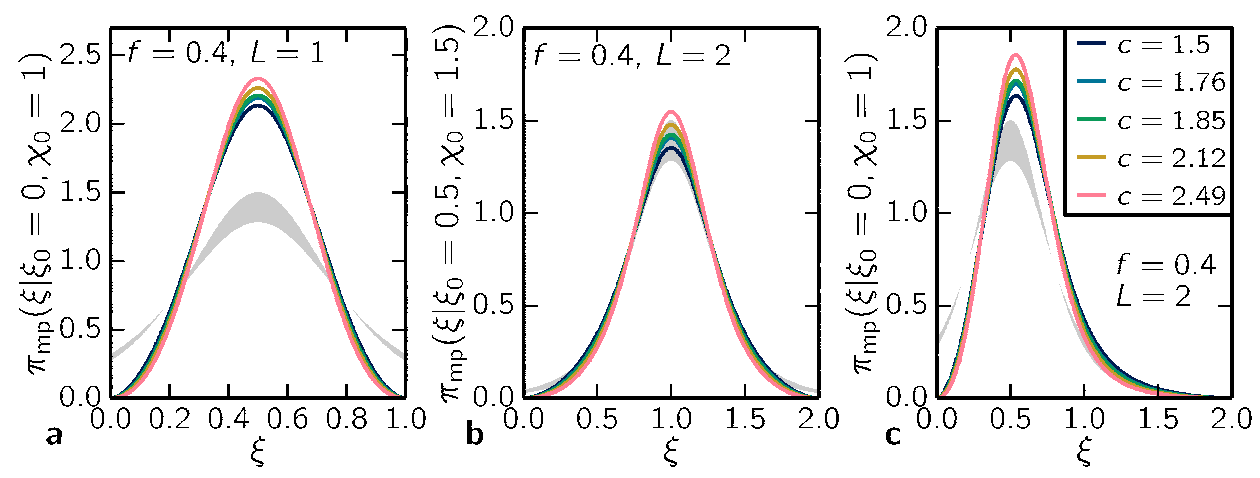

Figure 10. Meeting position - finite chain. Meeting position PDF for several values of $c$ for $f=0.4$ for different initial conditions: a) $L=1, \xi_{0}=0$ and $\chi_{0}=1$, b) $L=2, \xi_{0}=0.5$ and $\chi_{0}=1.5$. The PDF rapidly approaches the infinite chain value in case of the symmetric initial placement of the bubble. c) $L=2, \xi_{0}=0$ and $\chi_{0}=1$. The presence of the boundary modifies the shape of the distribution, which becomes more concentrated around the peak values. The shaded area represents the range of infinite chain values for identical parameters.

\section{Possible experimental verifications}

Successful experimental observation requires careful choice of the initial bubble size and the measurement temperature. Increasing temperature introduces more experimental noise into the measurements. In heteropolymers, the subdiffusive regime appears at temperatures close to the melting temperature. On the other hand, at low temperatures, the entropic effects play a reduced role.

The force $f=\beta \Delta G_{\mathrm{bp}} / 2$ resulting from the Gibbs free energy can be expressed in terms of the enthalpy and entropy differences

$$
f=\frac{\Delta H(T)-T \Delta S(T)}{2 k_{B} T} .
$$

At critical temperature $\Delta H\left(T_{c}\right)=T_{c} \Delta S\left(T_{c}\right)$; we linearly approximate the drift term

$$
f=\frac{\Delta S\left(T_{c}\right)\left(T_{c}-T\right)}{2 k_{B} T}=\frac{\Delta S^{\circ}\left(T_{c}\right)\left(T_{c}-T\right)}{2 R T} .
$$


( $R=k_{B} N_{A} \doteq 8.31 \mathrm{~J} /(\mathrm{K} . \mathrm{mol})$ is the gas constant and $N_{A}$ the Avogadro number). An example DNA chain with average molar $\Delta H^{\circ}\left(T_{c}\right) \equiv N_{A} \Delta H\left(T_{c}\right)=30 \mathrm{~kJ} / \mathrm{mol}$ and $\Delta S^{\circ}\left(T_{c}\right) \equiv N_{A} \Delta S\left(T_{c}\right)=90 \mathrm{~J} /(\mathrm{K} . \mathrm{mol})$ has $T_{c}=333 \mathrm{~K}$. The force at physiological temperature is thus $f \simeq 0.4$.

Previous experimental studies have measured the auto-correlation function which can be related to the bubble survival probability. The auto-correlation function is an integral of the meeting time PDF. In the case of the meeting time PDFs, we treated the diffusion constant as a parameter which would first have to be determined independently or fitted. Instead, we focus on the meeting position PDF in discussing the possible experimental realisations. The meeting position PDF is independent of the exact value of the diffusion constant, as the time variable is integrated out.

The difference between various values of $c$ can be clearly discerned at the peak as opposed to the tails, underlining the importance of knowing the entire meeting position $\mathrm{PDF}$. The difference in the peak value is $2.8 \%$ between $c=2.12$ and $c=1.76$ for a bubble of initial size of ten base pairs (see Figure 11). For a bubble of initial size of 100 bp and $1000 \mathrm{bp}$, the difference reduces to $0.68 \%$ and $0.17 \%$, respectively. Our method can be extended to cases when the initial bubble size has a known distribution; this requires a convolution of the distribution with the Green function. An experimental observation would require a preparation of bubbles of sizes of tens of base pairs at physiological temperatures and subsequent observation of the histogram of their closing position with a precision of $10^{-2}$.

Moreover, our results show a rapid approach of the finite chain to the infinite chain results. Therefore, precise knowledge of the chain length should not be crucial and a chain only several times longer than the initial bubble size would be sufficient to obtain data described by the infinite chain PDFs.

The approach in this work reduces the three-dimensional conformational dynamics of the DNA chain to the effective one-dimensional model with entropic interactions. Such dimensional reduction appears leads to logarithmic terms in free energy in other models as well. The interplay with bending and twisting of the DNA chain in three dimensions may further influence the bubble dynamics - e.g., by formation of a bend in the bubble which prevents its closing [50]. Care would have to be taken to separate the closing dynamics as described here from such additional events. For example, a light stretching force would reduce the formation of bends.

\section{Conclusions}

We have presented a solution of the Fokker-Planck equation governing DNA bubble dynamics in the framework of the Poland-Scheraga model both for normal diffusion, as well as for its fractional counterpart describing the subdiffusive dynamics in heteropolymers.

Expressions for the meeting time and meeting position PDFs have been given in

the analytical form in the Laplace/Fourier image and numerically transformed back to 


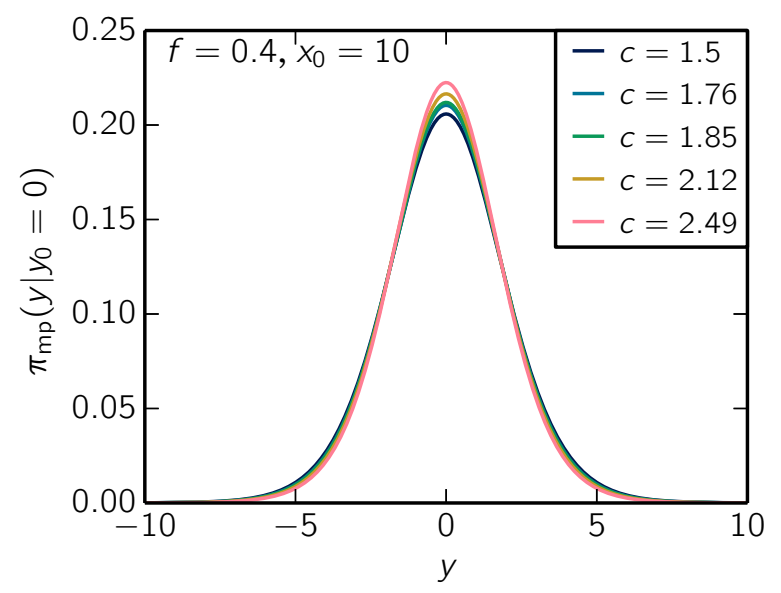

Figure 11. Possibility of experimental observation. For a DNA homopolymer around physiological temperatures and initial bubble size of 10 base pairs, the relative difference between the peak values of the meeting position PDFs for $c=2.12$ and $c=1.76$ is $2.8 \%$.

the full time/position variable range, thus extending previous results focused mainly on the asymptotic behaviour of the meeting time PDF. Meeting position PDF, which is a promising newly suggested quantity for the determination of the value of the entropic loop exponent, shows in the subdiffusive case surprisingly universal asymptotic features which appear to be pertinent to a whole class of stochastic processes. Furthermore, we have studied the influence of a finite length of the DNA chain on the diffusive dynamic of a bubble and shown a generic (with the only exception for the symmetrically placed bubble at the critical point) exponentially fast convergence to the infinite length limit.

Our solution constitutes a reference point for more involved models or numerical simulations. The results are relevant for determining the value of the entropic loop exponent from the bubble breathing dynamics in future experiments.

\section{Acknowledgments}

We thank Prof. Ralf Metzler for useful discussions and hospitality during our visits to his group. V.K. thanks Jens Karschau and Malte Vogl for valuable remarks on the manuscript. This work was financially supported by the Czech Science Foundation through grant No. P205/10/0989 (T.N.).

\section{References}

[1] Tobias Ambjörnsson, Suman K. Banik, Oleg Krichevsky, and Ralf Metzler. Breathing dynamics in heteropolymer DNA. Biophysical Journal, 92(8):2674-2684, Apr 2007.

[2] Michel Peyrard. Biophysics: Melting the double helix. Nature Physics, 2(1):13-14, Jan 2006.

[3] Douglas Poland and Harold A. Scheraga. Phase transitions in one dimension and the helix-coil transition in polyamino acids. The Journal of Chemical Physics, 45(5):1456-1463, 1966. 
[4] Douglas Poland and Harold A. Scheraga. Occurrence of a phase transition in nucleic acid models. The Journal of Chemical Physics, 45(5):1464-1469, 1966.

[5] A. Dechant, E. Lutz, D. A. Kessler, and E. Barkai. Superaging correlation function and ergodicity breaking for Brownian motion in logarithmic potentials. Physical Review E, 85(5):051124, May 2012.

[6] Ori Hirschberg, David Mukamel, and Gunter M Schütz. Diffusion in a logarithmic potential: scaling and selection in the approach to equilibrium. Journal of Statistical Mechanics: Theory and Experiment, 2012(02):P02001, 2012.

[7] Andreas Hanke and Ralf Metzler. Bubble dynamics in DNA. Journal of Physics A: Mathematical and General, 36(36):L473, 2003.

[8] Grégoire Altan-Bonnet, Albert Libchaber, and Oleg Krichevsky. Bubble dynamics in doublestranded DNA. Phys. Rev. Lett., 90:138101, Apr 2003.

[9] Hans C. Fogedby and Ralf Metzler. DNA bubble dynamics as a quantum Coulomb problem. Phys. Rev. Lett., 98:070601, Feb 2007.

[10] Hans C. Fogedby and Ralf Metzler. Dynamics of DNA breathing: Weak noise analysis, finite time singularity, and mapping onto the quantum Coulomb problem. Phys. Rev. E, 76:061915, Dec 2007.

[11] A Bar, Y Kafri, and D Mukamel. Dynamics of DNA melting. Journal of Physics: Condensed Matter, 21(3):034110, 2009.

[12] Tobias Ambjörnsson, Suman K. Banik, Oleg Krichevsky, and Ralf Metzler. Sequence sensitivity of breathing dynamics in heteropolymer DNA. Physical Review Letters, 97(12):128105, Sep 2006.

[13] Jae-Hyung Jeon, Pyeong Jun Park, and Wokyung Sung. The effect of sequence correlation on bubble statistics in double-stranded DNA. The Journal of Chemical Physics, 125(16):164901, 2006.

[14] Srijeeta Talukder, Pinaki Chaudhury, Ralf Metzler, and Suman K. Banik. Determining the DNA stability parameters for the breathing dynamics of heterogeneous DNA by stochastic optimization. The Journal of Chemical Physics, 135(16):165103, 2011.

[15] Craig J. Benham and Chengpeng Bi. The analysis of stress-induced duplex destabilization in long genomic DNA sequences. Journal of Computational Biology, 11:519-543, 2004.

[16] Jae-Hyung Jeon, Jozef Adamcik, Giovanni Dietler, and Ralf Metzler. Supercoiling induces denaturation bubbles in circular DNA. Phys. Rev. Lett., 105:208101, Nov 2010.

[17] Jie Ma, Lu Bai, and Michelle D. Wang. Transcription under torsion. Science, 340(6140):15801583, Jun 2013.

[18] Graeme A. King, Peter Gross, Ulrich Bockelmann, Mauro Modesti, Gijs J. L. Wuite, and Erwin J. G. Peterman. Revealing the competition between peeled ssDNA, melting bubbles, and SDNA during DNA overstretching using fluorescence microscopy. Proceedings of the National Academy of Sciences, 110(10):3859-3864, Mar 2013.

[19] Florian C. Oberstrass, Louis E. Fernandes, Paul Lebel, and Zev Bryant. Torque spectroscopy of DNA: Base-pair stability, boundary effects, backbending, and breathing dynamics. Physical Review Letters, 110(17):178103, Apr 2013.

[20] Michael E. Fisher. Effect of excluded volume on phase transitions in biopolymers. The Journal of Chemical Physics, 45(5):1469-1473, 1966.

[21] Yariv Kafri, David Mukamel, and Luca Peliti. Why is the DNA denaturation transition first order. Phys. Rev. Lett., 85:4988-4991, Dec 2000.

[22] Ralf Blossey and Enrico Carlon. Reparametrizing the loop entropy weights: Effect on DNA melting curves. Phys. Rev. E, 68:061911, Dec 2003.

[23] Lian-Ao Wu, Stephen S. Wu, and Dvira Segal. Looking into DNA breathing dynamics via quantum physics. Physical Review E, 79(6):061901, Jun 2009.

[24] Terence Hwa, Enzo Marinari, Kim Sneppen, and Lei-han Tang. Localization of denaturation bubbles in random DNA sequences. Proceedings of the National Academy of Sciences, 100(8):4411-4416, 2003. 
[25] D. J. Bicout and E. Kats. Bubble relaxation dynamics in double-stranded DNA. Physical Review E, 70(1):010902, Jul 2004.

[26] Tomáš Novotný, Jonas Nyvold Pedersen, Tobias Ambjörnsson, Mikael Sonne Hansen, and Ralf Metzler. Bubble coalescence in breathing DNA: Two vicious walkers in opposite potentials. EPL (Europhysics Letters), 77(4):48001, 2007.

[27] Jonas Nyvold Pedersen, Mikael Sonne Hansen, Tomáš Novotný, Tobias Ambjörnsson, and Ralf Metzler. Bubble merging in breathing DNA as a vicious walker problem in opposite potentials. The Journal of Chemical Physics, 130(16):164117, 2009.

[28] Y. Kafri, D. Mukamel, and L. Peliti. Melting and unzipping of DNA. The European Physical Journal B - Condensed Matter and Complex Systems, 27:135-146, 2002.

[29] John SantaLucia and Donald Hicks. The thermodynamics of DNA structural motifs. Annual Review of Biophysics and Biomolecular Structure, 33(1):415-440, 2004.

[30] D. Mukamel. Statistical mechanics of systems with long-range interactions. In Thierry Dauxois, Stefano Ruffo, and Leticia F. Cugliandolo, editors, Long-Range Interacting Systems: Lecture Notes of the Les Houches Summer School, volume 90. Oxford University Press, 2010.

[31] Hannes Risken. Fokker-Planck equation: Methods of Solution and Applications. Springer-Verlag, Berlin, 2nd edition, 1989.

[32] Frank W. J. Olver, Daniel W. Lozier, Ronald F. Boisvert, and Charles W. Clark. NIST Handbook of Mathematical Functions. Cambridge University Press, Cambridge, 2010.

[33] Sidney Redner. A Guide to First-Passage Processes. Cambridge University Press, 2001.

[34] Tomáš Novotný and Petr Chvosta. Resonant activation phenomenon for non-Markovian potentialfluctuation processes. Physical Review E, 63(1):012102, Dec 2000.

[35] Joseph Abate and Ward Whitt. A unified framework for numerically inverting Laplace transforms. INFORMS J. on Computing, 18(4):408-421, January 2006.

[36] Bruno Hupper and Eli Pollak. Numerical inversion of the Laplace transform. The Journal of Chemical Physics, 110(23):11176-11186, 1999.

[37] Harald Stehfest. Algorithm 368: Numerical inversion of Laplace transforms [D5]. Commun. ACM, 13(1):47-49, January 1970.

[38] Andreas Hanke, Martha G. Ochoa, and Ralf Metzler. Denaturation transition of stretched DNA. Physical Review Letters, 100(1):018106, Dec 2008.

[39] D. Marenduzzo, E. Orlandini, F. Seno, and A. Trovato. Different pulling modes in DNA overstretching: A theoretical analysis. Physical Review E, 81(5):051926, May 2010.

[40] Thomas R. Einert, Paul Näger, Henri Orland, and Roland R. Netz. Impact of loop statistics on the thermodynamics of RNA folding. Physical Review Letters, 101(4):048103, Jul 2008.

[41] William H. Press, Saul A. Teukolsky, William T. Vetterling, and Brian P. Flannery. Numerical Recipes: The Art of Scientific Computing. Cambridge University Press, Cambridge, 3rd edition, 2007.

[42] Ralf Metzler and Joseph Klafter. The random walk's guide to anomalous diffusion: a fractional dynamics approach. Physics Reports, 339(1):1 - 77, 2000.

[43] Ralf Metzler and Joseph Klafter. The restaurant at the end of the random walk: recent developments in the description of anomalous transport by fractional dynamics. Journal of Physics A: Mathematical and General, 37(31):R161, 2004.

[44] William Feller. An Introduction to Probability Theory and Its Applications. John Wiley \& Sons, 1971.

[45] Rhonald C. Lua and Alexander Y. Grosberg. First passage times and asymmetry of DNA translocation. Physical Review E, 72(6):061918, Dec 2005.

[46] S. Condamin, O. Bénichou, and J. Klafter. First-passage time distributions for subdiffusion in confined geometry. Physical Review Letters, 98(25):250602, Jun 2007.

[47] Vladimir V Palyulin and Ralf Metzler. Speeding up the first-passage for subdiffusion by introducing a finite potential barrier. Journal of Physics A: Mathematical and Theoretical, 47(3):032002, 2014. 
[48] Michael E. Fisher. Walks, walls, wetting, and melting. Journal of Statistical Physics, 34:667-729, 1984. 10.1007/BF01009436.

[49] Alan J Bray and Karen Winkler. Vicious walkers in a potential. Journal of Physics A: Mathematical and General, 37(21):5493, 2004.

[50] T. R. Strick, V. Croquette, and D. Bensimon. Homologous pairing in stretched supercoiled DNA. Proceedings of the National Academy of Sciences, 95(18):10579-10583, Sep 1998. 